\title{
Blockchain out of the Box - Where is the Blockchain in Blockchain-as-a-Service?
}

\author{
Alan Kernahan \\ IT University of Copenhagen \\ apaa@itu.dk
}

\author{
Ulrik Bernskov \\ IT University of Copenhagen \\ ulrb@itu.dk
}

\author{
Roman Beck \\ IT University of Copenhagen \\ beck@itu.dk
}

\begin{abstract}
Blockchain-as-a-Service (BaaS) is increasingly discussed as a way for companies to get started with blockchain projects. Different BaaS offerings are available, but a systematic categorization of what Baas comprises is missing. In this research, we analyze the service offerings of BaaS providers based on available online information and identify a number of common characteristics in the BaaS offerings related to the use of service types, distributed ledger technology (DLT) systems, consensus mechanisms, and pricing models. These characteristics are then further analyzed in the light of available literature on BaaS, as well as conducted expert interviews. The objective of this research is to provide an overview of the BaaS landscap,e as well as a taxonomy that provides guidance for researchers and practitioners alike interested in BaaS.
\end{abstract}

\section{Introduction}

Blockchain became known as the underlying technology behind the cryptocurrency Bitcoin [26]. Nowadays, distributed ledger technologies (DLT) are regarded as high-potential platform solutions that may disrupt traditional business models by reengineering supply networks and enforcing business logics and systemwide data integrity [6]. Initially designed to support a decentralized infrastructure that overcomes the need for central market agents, the disruptive nature of DLT systems swiftly garnered attention from a growing business community interested in applying blockchain technology in a range of applications and industries [43]. The originally permissionless, decentralized technology has been modified to support permissioned and distributed blockchain systems more suitable for commercial applications. These blockchain systems are intended to help enterprises to develop digital business models in networks, while keeping governance and thus control in a distributed, but not necessarily decentralized manner $[4,9,26]$. As a result, IT service providers are now offering Blockchain-as-a-Service (BaaS) upon which clients can develop and use blockchain applications [24]. According to Singh \& Michels [36], BaaS entails a service provider supplying and managing aspects of a DLT infrastructure to facilitate and bring efficiencies regarding the development, experimentation, deployment, and the ongoing management of DLT applications. However, it remains unclear how a technology that mainly gains its reliability from decentralization can be provided by a (central) IT service provider.

Blockchain-as-a-Service is marketed as a way to make DLT systems more accessible for enterprises with less overhead for adoption and at reduced costs [36]. With the rise of BaaS and its promises, there is also a rise in voices fearing that BaS providers are using blockchain to leverage selling digital transformation projects while creating a new dependency and centralization on the side of the BaaS provider [32]. In this research, we analyze the service offerings of BaaS providers and develop a taxonomy. More specifically, this paper will answer the following research question: What is Blockchain-as-a-Service, what does the current landscape of BaaS-providers look like and how can an overview help practitioners and researchers to navigate this new and emerging field of BaaS?

The remainder of the paper is structured as follows. In section two we will provide some literature background focusing on blockchain providers and BaaS topics related to the subject at hand. The third section introduces the methodological approach that we followed for collecting and analyzing data necessary to build a BaaS taxonomy, which will be elaborated on in section four. The fifth section presents a discussion of our main findings, while the paper concludes with a summary, critical discourse of our research, and future research directions in section six. 


\section{Related research}

Blockchain can be understood as a technology that allows for engineering markets [29]. Notheisen, Hawlitschek, \& Weinhardt [29] propose a blockchain market engineering framework that can be applied on a DLT or based upon community or application. The purpose of their framework is to provide a tool that allows for analyzing and subsequently designing the elements required for a functioning blockchain-based market. They distinguish between an environment layer, an infrastructure layer, an application layer, and an agent layer to "support the identification of applications and areas, in which blockchain-based economic systems offer effective and efficient solutions" [29, p. 1072].

The environment layer, or macro layer, of the market framework, comprises legal, social, and economic constraints, based on the applications, legal requirements, and the objects being transacted on the blockchain. The infrastructure layer covers both the setup of the protocol layer, which handles the blockchain ITinfrastructure, as well as the underlying hardware layer supporting the blockchain. More specifically, the protocol layer comprises the setup of the distributed ledger, the choice of consensus mechanism, as well as the cryptographic protocol, which enables the implementation of decentralized applications and economic tokens [29]. The provided blockchain market engineering framework provides a useful starting point to investigate the services and features provided by BaaS vendors.

While the technical stack of BaaS solutions supporting blockchain markets is one key area of interest, another is the organization and design of the governing body controlling BaaS solutions, as well as based upon markets, communities, and applications. More specifically, the distribution of decision rights, accountabilities, and incentives are of interest in distributed or even polycentric DLT systems [39].

Decision rights determine to what extent decisionmaking power is centralized (e.g., in a small group or a single entity) or decentralized (e.g., dispersed over many entities or people). This is typically determined by possession or ownership rights, but in DLT systems ownership can be hard to determine [7]. However, in a BaaS environment, ownership is clearly defined on the infrastructure layer, as well as on the application layer.

Accountability is enacted "specified and brought into force, through contracts and legal frameworks governed by institutions" [7, p. 1023], and enforcement mechanisms are essential. While the enactment of governance in most DLT-systems is on-chain, where accountability can rest with several entities in a distributed or even decentralized system, in BaaS, ac- countability is organized in a similar way as an outsourcing or cloud usage contract.

Incentives motivate entities in a system to act in a desired, predetermined way. Typically, a differentiation is made between pecuniary incentives, which relate observable agent behavior to monetary rewards and non-pecuniary incentives, which relate observable agent behavior to nonmonetary rewards, such as privileges, visibility, or reputation. In the case of BaaS, the incentives are defined by service contracts.

Finally, one of the most visible forms of enacted governance in DLT systems is forking, which can create different DLT species, adaptations, or variations [3]. Here again, the question emerges if, and how forking is possible in BaaS environments.

\section{Research methodology}

\subsection{Developing a taxonomy, a bricolage of dif- ferent approaches}

As the area of BaaS and related service offerings is still at an early stage, we adopt a bottom-up approach, where categories emerge from inductive empirical observations. A useful taxonomy comprises a classification of the entities analyzed, e.g., by relating them to each other and placing them in a wider, meaningful context and provides an insightful description of the entities (something that in biological classification work is called 'diagnosis'), its main characteristics, how these are employed, and where.

Methodological approaches to the development of taxonomies are sparse within information systems research. However, we took guidance from Nickerson, Varshney and Muntermann [28], who present a generalized method to the development of taxonomies and how to apply them in information systems research. They state that "the development of a taxonomy involves determining the characteristics of the objects of interest. The choice of the characteristics in a taxonomy is a central problem in taxonomy development" [28, p, 343]. In order to avoid choosing characteristics in a naïve way, one has to define meta-characteristics in the beginning stages of a taxonomy development process, so that these may serve as a basis of the identification of the more general characteristics in the taxonomy.

While these meta-characteristics ideally should be developed at the start of the development process of the taxonomy, Nickerson et al. found "that the metacharacteristic sometimes does not become clear until part way through the taxonomy development process" [28, p, 343]. Thus, to mitigate the risk of choosing meta-characteristics prematurely, we followed the guid- 
ance from research on blockchain layers and market engineering, as well as governance structures to get started with our taxonomy, while still allowing for empirical observations to influence the sense-making of them. Doing so is also in line with the chosen analysis approach inspired by Gerber, Baskerville, and Van der Merwe [16], where we continuously questioned the validity of the overall meta-characteristics, as the number and nature of our empirical observations increased. Nickerson et al. [28] postulate that because the development of meta-characteristics is an iterative method, one must determine ending conditions for when this iterative method should cease. The next step of the analysis entails making a conscious choice between proceeding with either an "empirical-to-conceptual approach" or a "conceptual-to-empirical approach", including the subsequent steps pertaining to that approach. We applied a hybrid-form of these two approaches in the development of our taxonomy, as it became clear that the model illustrating this method of taxonomy development would not be useful as a framework for our approach. However, certain aspects should be taken into consideration, i.e. the metacharacteristics and some of the ending conditions described above.

Apart from inspirations from Gerber et al. [16] taxonomy of classification approaches (ToCA) and aspects of Nickerson et al. [28] method of taxonomy development, we also took guidance from prior taxonomies on blockchain to build upon their work. More specifically, we were inspired by Labazova, Dehling and Sunyaev's [23] taxonomy of blockchain applications, both in the incorporation, as well as in the defining of, our meta-characteristics (e.g. Consensus Mechanism).

Thus, our research methodology is a bricolage of these methods [16, 28, 41], which is an approach that we regard as suitable, as it allows the combining of different instruments and methods in a new and emerging research field, where established best practices are not yet clearly defined.

\subsection{Data collection}

Because of the new and emergent nature of the field of BaaS, we decided to collect our empirical data following an online desk research approach, where we applied modulated searches on the Internet, following the recommendations provided by Bryman [10]. For our research on different search engines such as google.com and bing.com we used different keywords and Boolean combinations of them, e.g., "Blockchainas-a-Service", "BaaS", "service", "service providers", "blockchain services", "DLT systems", "DLT services", "blockchain cloud", among others. After sever- al search iterations and focus on BaaS providers and not on articles or blogs just discussing BaaS, we initially identified $26 \mathrm{BaaS}$ providers. However, since most articles and blogs did not provide enough technical details on the BaaS solutions which we considered mandatory (e.g., service types, pricing models), we eventually settled on 15 BaaS providers in total, which we then analyzed further.

In addition to our online search, we conducted two semi-structured interviews with the heads of blockchain from two major consulting companies to obtain a greater depth of information regarding the topic at hand [22], and to make sure that we identified the relevant BaaS providers. These interviewees were selected by availability and willingness to participate in our research, but also due to the fact that consultancies are exposed to many different industries, and thus have a more general overview of how $\mathrm{BaaS}$ is emerging across industries. The interviews also helped us in the formation of the meta-characteristics for our taxonomy, as we were able to integrate insights from the two experts regarding characteristics and strategies behind the different BaaS solutions into our research. From the interviews, we were able to identify that trust enabled blockchain market platform features and interoperability as important characteristics. The interviews lasted about 30 minutes each and were recorded and transcribed.

\subsection{Data analysis}

Due to the new and emerging nature of BaaS, no industry acknowledged terminology has been sufficiently established yet, which is illustrated by the wide variety of diverging terms to explain the same concepts. For example, the terms "platform", "protocol" and "blockchain core" are often used interchangeably to describe the underlying DLT system (e.g., Ethereum or Hyperledger Fabric) on which the BaaS providers run their service, while others use the term "platform" to describe the software, which interacts with the underlying DLT system.

The lack of a commonly used vocabulary or defined terms has been one of the motivations to develop a taxonomy that provides guidance and structure. Thus, in the first iteration of the analysis approach, we defined general meta-characteristics, such as "platform" (at this point, we used the term platform, referring to the specific DLT system used), "consensus mechanism", "pricing model", "performance", "scalability" and "interoperability".

Additionally, we also pre-defined some categories based on our literature research, such as "Blockchainas-a-Service", "Platform-as-a-Service" and "Infrastructure-as-a-Service". However, over the course of several 
iteration cycles preluded by discussions within the research group, we concluded that the categorization division of infrastructure-, platform-, or application-asa-service did not serve well to illustrate the BaaS landscape, as most vendors actually offered a combination of these, if not all three. Consequently, we converted these three distinct service offerings into subcharacteristics to effectively classify the different service types.

Another issue in the data analysis has been the different levels and granularity of information provided by the BaaS providers. We defined a minimum requirement of technical details for a provider to be included in our overview. For example, for some providers, it was unclear from their websites or white papers, which service types they were offering, and as such, they were excluded from the analysis. However, when it came to the meta-characteristic of pricing models, we did not choose to be as stringent, and included providers who did not disclose their specific pricing model in our analysis. This was because even though pricing models are an important and interesting differentiator, it is not deemed as essential for the overview as the technical details. Another requirement was that the technical information needed also was available in a language we could understand. For example, some of the Chinese BaaS providers only had technical details available in Mandarin, which presented a challenge. However, even after a translation through online translation services, the translated information did not make enough coherent sense to be included in the taxonomy, whereas other translations did. Furthermore, we originally also analyzed blockchain services from consul- tancies. However, since we were not able to find any tangible information on their BaaS offerings, these were excluded from our taxonomy as well.

We decided to end the recurrent iterations of the taxonomy, based on the chosen objective ending conditions of 1) having classified at least one object under every characteristic of every dimension, 2) that no dimensions or characteristics were split or merged in the last iteration and 3) that we had not added any new dimensions in the last iteration [28].

\section{Taxonomy of BaaS services}

In this chapter, we will present the results of our taxonomy of BaaS service types, divided into infrastructure layer services, protocol layer services, and application layer services. Subsequently, we will compare the different consensus mechanisms supported by the different BaaS service providers before we will provide BaaS pricing model characteristics. While the concept of BaaS generally involves some sort of platform from where customers will be able to make use of the underlying blockchain protocol, there are variations as to how the providers manage the infrastructure of the service, and which types of applications are offered on their platforms.

\subsection{Infrastructure layer service characteristics}

One of the infrastructure possibilities, which we found common for many of the providers, includes the offer of running the blockchain platform on hardware

Table 1. A taxonomy of Blockchain-as-a-Service characteristics.

\begin{tabular}{|c|c|c|c|c|c|c|c|c|c|c|c|c|c|c|c|c|c|}
\hline \multirow[b]{2}{*}{$\begin{array}{l}\text { BaaS Service } \\
\text { Provider }\end{array}$} & \multicolumn{2}{|c|}{ Infrastructure } & \multicolumn{2}{|r|}{ Protocol } & \multicolumn{2}{|c|}{ Application } & \multicolumn{5}{|c|}{ Consensus Mechanism } & \multicolumn{6}{|c|}{ Pricing Model } \\
\hline & Own & $\begin{array}{c}\text { 3rd } \\
\text { party }\end{array}$ & $\begin{array}{l}\text { Single } \\
\text { DLT }\end{array}$ & Multiple DLTs & $\begin{array}{l}\text { Pre- } \\
\text { made }\end{array}$ & $\begin{array}{c}\text { Development } \\
\text { tools }\end{array}$ & PoW & BFT & CFT & PoS & other & $\begin{array}{c}\text { Trans- } \\
\text { actions }\end{array}$ & \begin{tabular}{|c|} 
Per \\
node
\end{tabular} & \begin{tabular}{|c|} 
Stor- \\
age
\end{tabular} & $\begin{array}{c}\text { Number } \\
\text { of IP }\end{array}$ & $\begin{array}{l}\text { CPU } \\
\text { use }\end{array}$ & N/A \\
\hline Alibaba & $X$ & - & - & HF, Eth, other & $X$ & - & - & $x$ & $x$ & - & - & - & - & $X$ & - & - & - \\
\hline Amazon & $x$ & - & - & HF, Eth, other & $x$ & $x$ & - & $x$ & $x$ & - & - & $x$ & $x$ & $x$ & - & - & - \\
\hline Huawei & $X$ & - & $\mathrm{HF}$ & - & $x$ & - & - & - & $X$ & - & - & $x$ & - & - & - & - & - \\
\hline SAP & $x$ & - & - & HF, other & $x$ & $x$ & - & $x$ & $x$ & - & - & - & - & $x$ & - & $x$ & - \\
\hline Kaleido & - & $x$ & Eth & - & $x$ & $x$ & - & $x$ & - & - & - & - & - & - & - & - & $x$ \\
\hline Oracle & $X$ & - & $\mathrm{HF}$ & - & $X$ & $x$ & - & - & $X$ & - & - & $X$ & $\cdot$ & - & - & - & - \\
\hline Tencent & $x$ & - & own & - & - & $x$ & $x$ & $x$ & - & - & - & - & - & - & - & - & $x$ \\
\hline IBM & $X$ & $x$ & $\mathrm{HF}$ & - & - & $X$ & - & - & $x$ & - & - & - & - & $X$ & $X$ & $X$ & - \\
\hline Microsoft & $x$ & - & Eth & - & - & $x$ & - & $x$ & - & - & - & $x$ & $x$ & $x$ & - & - & - \\
\hline Hewlett-Packard & $x$ & - & R3 & - & - & - & - & $x$ & - & - & - & - & - & - & - & - & $x$ \\
\hline Baidu & $X$ & - & - & HF, Eth, own & - & - & - & $X$ & $X$ & - & $X$ & - & - & - & - & - & $X$ \\
\hline Block.one & $x$ & - & other & - & - & $x$ & - & - & - & $x$ & - & - & - & - & - & - & $x$ \\
\hline Stratis & - & $x$ & Bit & - & - & $x$ & $x$ & $x$ & - & $x$ & - & - & $\cdot$ & - & - & - & $X$ \\
\hline Samsung & $X$ & $x$ & - & HF, Eth, own & $X$ & $X$ & $X$ & $X$ & $X$ & - & $X$ & - & - & - & - & - & $X$ \\
\hline Chainstack & - & $x$ & - & Eth, R3, Bit, other & - & $x$ & $X$ & $X$ & - & - & - & - & $X$ & $X$ & - & - & - \\
\hline
\end{tabular}


provided by the vendor. This setup normally includes hosting the blockchain in a cloud solution offered by the provider. One more often finds such a setup offered by larger IT service providers, since they already have the cloud infrastructure in place.

The other infrastructure possibility, which we found in a few of the services, is to host the BaaS on a third-party infrastructure. One example of this is Kaleido [21], who offer their customers to set up their platform on either Amazon or Microsoft Azure cloud. A few providers, such as IBM and Samsung [19, 34], also offer the possibility to choose between either hosting the blockchain in their own cloud or in a thirdparty cloud.

\subsection{Protocol layer service characteristics}

Blockchains and DLTs in general are distributed data management systems stored on different servers or nodes. Generally, all full nodes in the network will receive a replication of all the transactions in the network for storage. In permissioned BaaS systems, assigned validator nodes have the responsibility of appending and validating new blocks [14]. However, when analyzing the different BaaS solutions, we found that many providers offer not just one, but several DLT systems with different consensus properties.

The most commonly offered DLT system is Hyperledger Fabric, and providers such as Huawei, Oracle, and IBM are offering BaaS focusing solely on the use of Hyperledger Fabric as the underlying platform protocol [19, 31]. Hyperledger Fabric is a modular blockchain platform, which enables the possibility of using the platform in a variety of different use cases and industries, and it is governed by IBM and the Linux foundation [4]. Furthermore, Hyperledger Fabric operates in a permissioned public environment, which means that new members interested in joining the network must be selected and approved in advance. Thus, access is restricted to approved network participants only. This makes it especially useful for enterprise blockchain solutions, as our BaaS expert interviews also confirmed.

The other frequently supported DLT protocol is a permissioned version of Ethereum. The original version of Ethereum is a permissionless public protocol that focuses on providing a platform, where anyone can "write smart contracts and decentralized applications, where they can create their own arbitrary rules for ownership, transaction formats and state transition functions" [11]. As such, the aim of Ethereum is to provide a generic platform for different kinds of applications and transactions. Several of the blockchain providers we identified are using a permissioned version of Ethereum, called Quorum, which offers to pro- vide data privacy to transactions and smart contracts in DLT networks [26]. BaaS providers focusing solely on the use of permissioned versions of Ethereum, include Microsoft Azure and Kaleido [21, 25].

Hyperledger Fabric and Ethereum are not the only DLT protocols offered for BaaS. Another protocol that we identified that is being offered is R3's Corda [9], which is a permissioned public DLT protocol focusing on applications mainly in the financial services industry. Some providers also offer CoinSciences' MultiChain [17], which is a fork of the Bitcoin protocol that aims to provide permissioned blockchains, which can be used by organizations for financial transactions. Others offer their own proprietary protocols, while also aiming to meet greater privacy, throughput, and response times for customers within different areas of application [33, 34, 38]. The only identified providers offering permissionless public services are Stratis and Chainstack [13, 37], in addition to permissioned versions of the different protocols.

Six of our 15 BaaS service providers offer more than one DLT protocol, while five support either Ethereum or Hyperledger Fabric, and four either Bitcoin, Corda R3, or something else altogether. Alibaba and Samsung offer the choice between one or two different DLT protocols, mainly Hyperledger Fabric or a permissioned version of Ethereum, while Baidu claims to offer three different DLT protocols [1, 33, 34].

A BaaS vendor that we identified as particularly interesting in relation to offering support to a wide variety of DLT protocols is Chainstack. They claim to offer Corda R3, Quorum, MultiChain, Ethereum and the Bitcoin protocol. At the same time, they also offer the opportunity to create and maintain blockchain networks in Google Cloud, Amazon Cloud, Azure Cloud, Alibaba Cloud, and others [13]. Therefore, they are the only provider offering an infrastructure independent BaaS offering that supports more than one DLT protocol.

\subsection{Application layer service characteristics}

One of the main differences that we found in the blockchain services offered is that there is a lot of variation in the setup of the application layer. Specifically, the different applications that are offered and what they can do for the customer. Some of the larger blockchain providers, such as Alibaba, Amazon, Huawei, Oracle and Samsung use their platforms to provide predefined or generic applications that are ready to be used by their customers. Some of the offered applications include operations management, smart contract management and node management, related to managing the blockchain network $[1,2,18,34]$. However, some 
providers also claim to offer pre-made applications such as supply chain tracking, digital identity authentication and cash flow tracing, amongst other services that can be integrated in the cloud $[18,31]$.

One commonality we identified amongst several service providers is that many of the platforms offer a variety of different applications with tools to ease the use of developing own applications or networks on the platform. This is especially the case within the IBM Blockchain Platform and Microsoft Azure Workbench $[19,25]$. Other providers such as Block.one and Stratis provide similar application development tools, but instead use their own, or the Bitcoin protocol, to run their platforms [8, 37].

Many of the larger providers, such as SAP, Alibaba, Oracle, Amazon and others seem to specifically focus on the capability of providing the infrastructure, platform and applications needed in their service, as full-stack services. Thus, by providing a combined front-end and back-end solution, they offer BaaS as a standardized solution in extension to their existing cloud services on their platform $[1,2,31,35]$.

\subsection{BaaS consensus mechanism characteristics}

Since Hyperledger Fabric and permissioned versions of Ethereum are the most commonly offered BaaS protocols, the consensus algorithms commonly used for those platforms were also the ones most frequently identified in our taxonomy. However, even though we identified every BaaS provider, except one, to use some form of Byzantine Fault Tolerance (BFT) or Crash Fault Tolerance (CFT) consensus mechanism, we identified that the vendors offered the use of several different variations of BFT and CFT [12].

Hyperledger Fabric is a modular blockchain protocol, which is also the case when choosing a consensus mechanism. We identified that currently the most used consensus mechanisms for BaaS offered by $\mathrm{Hy}$ perledger Fabric are Kafka [20] and Raft [30], which are Crash Fault Tolerant (CFT) ordering services. In the CFT setup, a leader node is appointed and is assumed to act honestly. Since all the other nodes will replicate the leader node's entries, it is necessary to trust the leader. However, since the CFT consensus or ordering service is only used in permissioned networks, it is assumed that leaders can be trusted. If the leader crashes, a new leader will take its place [42]. However, the centralized concept of the CFT algorithm also enables increased speed in the consensus process by using transaction finality. We identified Alibaba, Amazon, Huawei, Oracle, SAP, IBM and Samsung to offer Kafka CFT on Hyperledger Fabric, while only Baidu offers Raft. MultiChain uses a similar consensus algorithm to Hyperledger Fabric, however, the leader updating the blockchain is chosen in a round-robin or polled fashion, depending on their historical contributions to the blockchain [17]. The MultiChain version of BFT is offered by SAP [35].

Like Hyperledger Fabric, Quorum is compatible with Raft, but it is also compatible with the BFT consensus variant called Istanbul BFT. Different from CFT algorithms, which use a leader or block proposer, the Istanbul BFT algorithm requires validators to perform multiple rounds of voting for each block. With this approach, the network can tolerate $1 / 3$ faulty nodes [42]. Thus, Istanbul BFT can be used in instances where competitors or other network participants cannot be trusted and is used by the majority of BaaS providers offering Quorum on their platforms, such as Alibaba, Microsoft Azure, Samsung, Chainstack and Kaleido. Other providers, such as Tencent, Stratis and Chainstack offer the possibility of using the Bitcoin protocol and maintain the network using a Proof of Work consensus algorithm [13, 37, 38].

Comparable to what we identified in relation to BaaS service type offerings and protocol offerings, it seems that most of the BaaS vendors provide the possibility to choose between different consensus algorithms, depending on the characteristics of the network that customers are seeking. Stratis, Chainstack and Tencent $[13,37,38]$ offer very different types of consensus mechanisms at the same time (such as Proof-ofWork and BFT), while all other vendors provide variations of BFT or CFT consensus mechanisms. Clearly, consensus mechanisms offered by BaaS providers focus on performance and speed in permissioned public networks. This is done by centralizing the consensus finding approach within the network.

\subsection{BaaS pricing models}

One of the common pricing models that we found to be offered by several blockchain providers, is that customers can pay based on the number of instances or transactions per hour. This pricing model is used by Amazon, Huawei, Oracle and Microsoft Azure [2, 18, $25,31]$. Another very common pricing model is to pay for the storage space needed, based on the number of peers in the network. Amazon, SAP, IBM, Microsoft Azure, and Chainstack [2, 13, 19, 25, 35] offer this pricing model with hourly rates, whereas Alibaba offers a monthly fee for the storage space used.

We also found that Amazon, Microsoft Azure and Chainstack charge based on the number of peer nodes in a network. At the same time, IBM and SAP offers the possibility to charge an hourly fee based on the CPU usage or workload of the servers, which indicates how frequently the blockchain networks are used. Fi- 
nally, we also found that IBM's pricing model includes a fee for IP allocation [19, 35].

One of the tendencies that we found in relation to the identified pricing models offered by BaaS providers is that almost every one of the providers offer several different pricing model possibilities. It was mainly the larger enterprise BaaS providers that had readily available pricing models, and most of their pricing models are focused on the costs related to maintaining the infrastructure of the blockchain network in the cloud. As such, the identified pricing models currently offered for BaaS solutions are like pricing models that exist for other cloud services that these vendors offer.

\section{Discussion}

Based on the results of our BaaS taxonomy and related literature, we discussed our findings with two senior blockchain consultants from two different large consulting companies to find out more about the status of the BaaS market, the relevance of the provided business services, as well as critically discuss governance and decentralization aspects of BaaS service offerings.

\subsection{Enterprise BaaS market}

As illustrated by the BaaS providers identified in our taxonomy, the current market for BaaS on an enterprise level is dominated by large IT service providers. Amazon, Microsoft and IBM "are the three predominant BaaS providers that are mentioned when I ask my customers". (Senior blockchain consultant B)

Our analysis revealed that the BaaS market is very new, and that it is characterized by providers trying to build their BaaS offerings on their existing cloud computing business models. Companies are still struggling to find their footing in the emerging DLT world, and an offering such as BaaS is something that sounds similar to other traditional service sourcing approaches such as cloud computing. Companies thus believe that BaaS is a potential way into blockchain. In so doing, enterprises do not have to build and maintain their own blockchain infrastructure and applications [32], at least that is how it appears to them. Cloud-based BaaS service offerings by large IT providers are often regarded as an extension of their existing business models. This was also echoed in the interview with our subject matter expert:

"If you look at SAP mainly - maybe also Microsoft and Oracle, but SAP they especially like to come up with use cases that will work in extension of their existing services". (Senior blockchain consultant A)
Consequently, BaaS providers are trying to make blockchain more readily available for their clients and easier to get started with. However, as we also found in our taxonomy, the characteristics of these blockchain services are not decentralized, as originally seen with Bitcoin and Ethereum, on the infrastructure level. Only very few BaaS providers allow for different IT infrastructures to be used. Most BaaS providers only allow their own cloud-based BaaS services, which makes these offerings highly centralized on the infrastructure layer. This can lead to a lock-in effect and single point of failure for a lot of clients, as they are bound to a single provider controlling the infrastructure. BaaS service providers may see this as their chance to stay in business and not be disintermediated, like other trusted agents could become, through the use of DLT:

"By controlling the technological infrastructure and offering something that looks like blockchain, BaaS service providers will get customers on their platform. So, I also think part of it is seeing it as a market opportunity to get into the market - and if you are not in the blockchain market, you will also not be able to keep up". (Senior blockchain consultant A)

We also found that several of the BaaS service providers use an approach where they offer the use of different DLT protocols with a variety of potential consensus mechanisms, for example, "SAP, Oracle and Microsoft have multi-strategy where you can choose between several different ledgers and tie them together." (Senior blockchain consultant A). This reduces the risk for clients to strand with one service provider, but as the market for BaaS is still very new, it is not yet clear how interoperability, if needed between different BaaS service providers, platforms, and consensus mechanisms, can be achieved across platform boundaries: "There has been a couple of projects on interoperability, but we are very far from reaching the goal of interoperability. We have not even reached blockchain mass adoption yet". (Senior blockchain consultant A)

However, once interoperability is an option, it may very well impact the current BaaS market, since that would make it possible for customers to use and interact with other platforms instead of settling on a single BaaS provider's platform.

\subsection{Blockchain services and economy}

Based on the BaaS characteristics identified in our taxonomy, BaaS is an example for the ongoing servitization like Software-as-a-Service, Infrastructure-as-aService, and so on. BaaS service providers seem to offer a mix of these services, and focus on making blockchain platforms easily available, as a service to assist customers in their own blockchain value-creation processes. BaaS generally entails a platform upon 
which customers can create their own networks, even though the infrastructure itself is mostly centralized.

From a decentralized economy perspective, the original intention was a decentralized distributed ledger that eliminates the need for an intermediary to reach consensus over transactions, in order to lower costs. However, at the infrastructure layer, BaaS services are largely centralized within permissioned platforms, and it therefore seems relevant to discuss what other economic advantages can be gained from the use of BaaS offerings instead?

One characteristic of blockchains is that they are distributed databases, and consequently, "anything configurable, or able to be represented in a digital database, can be on a blockchain" [15, p. 8]. Thus, BaaS service providers offer a variety of applications to create digital representations of physical things, to use the DLT as a repository overview of physical artefacts. "That's currently what we are seeing with track and trace solutions and with TradeLens and the documents related to it. Obviously, once there is a physical dimension to it, it is also more complex. So therefore, it is a general ongoing trend, which will continue". (Senior blockchain consultant A). Therefore, one economic incentive for using BaaS is to create digital processes of physical artifacts for better tracking and transparency.

Another economically interesting characteristic of blockchains in an enterprise context is that it enables the creation of smart contract-facilitated transactions, with the aim to lower information asymmetries within a network of business partners, e.g., in the process of issuing and settling invoices [15]. However, using smart contracts to facilitate transactions on a permissioned platform of a BaaS service provider is somewhat an over-engineered solution, as other centralized solutions exist that achieve the same without the need for putting a DLT system on top of a centralized infrastructure. "If you instead start to create the transactions uniquely and two-way signed in the middle, then you would do something that also works in an ERP system". (Senior blockchain consultant A)

Finally, DLT systems enable consensus without the need for centralized trust, which can lower transaction costs. However, what we identified is that many of the BaaS services still have a large degree of centralized trust through their choice of consensus mechanisms. The advantage of this setup is that it can be " $a$ way to control opportunism in the presence of bounded rationality and asset specificity by internalizing the transaction costs of opportunism" [15, p. 12]. Nevertheless, the newness of the BaaS market indicates that the current focus is more on providing ease of use, availability, and low entrance costs for customers, rather than on scalability.

\subsection{BaaS from a decentralized governance point of view}

From a decentralized governance perspective, which is the idea underlying the dissemination of power to not require a trusted third party, services based on a BaaS may in fact lead to less control over applications and the overall system [36]. As BaaS services can be contractually negotiated like any other outsourcing, BaaS services might be based on centralized infrastructure and heavily centralized governance. This is somewhat paradoxical, because clearly specified governance and accountability is so vital to their customers: "We see that the majority of the Fortune 500 can only use permissioned systems, primarily due to compliance issues". (Senior blockchain consultant B)

Risk averse customers must ensure that the networks they are using comply with regulation, and for them, BaaS may be seen like an attractive hybrid between operating a distributed network in a permissioned system, while the IT infrastructure is provided centrally, as they are used to. "There is regulation, there is compliance, there are laws... by law they [companies] are forced to do things like KYC (Know your customer), KYB (Know your business) where this conflicts with decentralized, completely permissionless networks". (Senior blockchain consultant B)

When it comes to governance and decision rights, then permissioned solutions are obviously more centralized than permissionless systems. This again is something companies are familiar with, as they are regulated entities and, as such, accountability is enacted much more on an institutional level, than a technical level [7]: "The conversation is not at the technology level anymore. It is at the governance level. In any enterprise, doing any kind of business, you need to have some boundaries, some kinds of control. A system, where everybody can do business as we do business today without any kind of control or boundaries, might not be ideal... that is not how we can do business". (Senior blockchain consultant B)

In line with this, identity management become core components of BaaS, due to identity and confidentiality aspects. Permissioned systems allow for restricting access to partners, where trust may already exist to some extent. "How do you get trust in a permissionless network?... Trust must happen at the protocol level because there is no entrance. Whereas in the permissioned world, trust can happen at the entrance level and hence you can gear the consensus mechanism for the intended use case". (Senior blockchain consultant B). Obviously, a permissioned DLT system is not only having a negative impact on the idea of decentralized governance, but also on the entire reasoning for having blockchain solutions in the first place. 


\section{Conclusion}

Current BaaS service offerings mostly focus on offering blockchain service solutions to companies that focus on characteristics such as performance, ease of use, and development on permissioned platforms. In this research, we presented an analysis of BaaS based on qualitative research in the form of interviews with two subject matter experts on the topic of BaaS services, as well as desk research on current BaaS providers' service offerings and their characteristics. The purpose of the paper was to explain what $\mathrm{BaaS}$ is and how it is related to the original idea of decentralization, provided by blockchain. As such, we provide a first conceptual contribution to the academic discourse on the emerging field of BaaS.

In our taxonomy, we analyzed the current state of the BaaS market, specifically regarding service characteristics, support of different DLT protocols and consensus mechanisms, and related pricing models for the service provisioning. Our findings illustrate that the BaaS market is dominated by large IT service providers that offer a variety of different, but centralized services. These findings might be in-line with the needs of corporate customers, who prefer permissioned DLT solutions, focusing on BaaS characteristics such as performance, ease of use and availability. This is mainly due to the need for clearly defined governance, decision rights, and accountabilities to stay legally compliant, which is assured by using BaaS. Our research provides guidance for organizations considering $\mathrm{BaaS}$ as a way to get started with blockchain to sensitize them for the pros and cons of BaaS solutions currently on the market. While our research and the taxonomy developed provides a first systematic overview and metacharacteristics of BaaS, it needs to be admitted that the research on $\mathrm{BaaS}$ is at the very beginning and our taxonomy might be limited, both due to the few BaaS solutions available, as well as the sometimes premature documentation of the offered solutions. Thus, we ask for revisiting our research once the BaaS offerings are more mature. Even though most of these blockchain services do not constitute a distributed, or even decentralized, network of independent DLT nodes, which provides the security that the network is not dominated by a single, potentially malicious acting agent, they might provide a gateway for customers to get started with DLT projects, like a safe playground that allows for building DLT competencies. More research is needed on the pros and cons of BaaS, especially if they turn out to be a stepping stone for companies to get started, or if they become a dead-end road with lockedin customers using cloud-based services that are not so different from more traditional offerings.

\section{References}

[1] Alibaba (2020). Blockchain as a Service https://www.alibabacloud.com/help/product/84950.htm?spm $=\mathrm{a} 2 \mathrm{c} 63 . \mathrm{p} 38356 \cdot \mathrm{a} 1.1 .104712 \mathrm{eckfFksS}$ [Accessed May 23 ${ }^{\mathrm{rd}}$, 2020]

[2] Amazon (2020). What is Amazon Managed Blockchain? https://docs.aws.amazon.com/managed-

blockchain/latest/managementguide/what-is-managed-

blockchain.html [Accessed May 23 ${ }^{\text {rd }}, 2020$ ]

[3] Andersen, J. V., \& Bogusz, C. I. (2019). Self-organizing in blockchain infrastructures: Generativity through shifting objectives and forking. Journal of the Association for Information Systems, 20(9), 11.

[4] Androulaki, E., Barger, A., Bortnikov, V., Cachin, C., Christidis, K., De Caro, A., ... \& Muralidharan, S. (2018, April). Hyperledger Fabric: A distributed operating system for permissioned blockchains. In Proceedings of the Thirteenth EuroSys Conference (pp. 1-15).

[5] Baidu (2020) Baidu Raft. https://github.com/baidu/braft [Accessed May 23 ${ }^{\text {rd }}, 2020$ ]

[6] Beck, R. (2018). Beyond Bitcoin: The rise of blockchain world. Computer, 51(2), 54-58.

[7] Beck, R., Müller-Bloch, C., \& King, J. L. (2018). Governance in the blockchain economy: A framework and research agenda. Journal of the Association for Information Systems, 19(10), p. 1020-1034.

[8] Block.one (2018). EOS.IO Technical whitepaper v.2. https://github.com/EOSIO/Documentation/blob/master/Tech nicalWhitePaper.md [Accessed May 23 ${ }^{\text {rd }}, 2020$ ]

[9] Brown, R. G., Carlyle, J., Grigg, I., \& Hearn, M. (2016). Corda: An introduction. R3 CEV, August, 1, 15.

[10] Bryman, Alan (2008). Social research methods. Oxford University press, Oxford.

[11] Buterin, V. (2013). Ethereum whitepaper. GitHub repository, 22-23.

[12] Castro, M., \& Liskov, B. (1999). Practical Byzantine Fault Tolerance. In Proceedings of the Third Symposium on Operating Systems Design and Implementation, New Orleans, USA, February 1999, (99), p. 173-186.

[13] Chainstack (2019). Chainstack docs. https://docs.chainstack.com/platform/supported-protocols [Accessed May 23 ${ }^{\text {rd }}, 2020$ ]

[14] Danzi, P., Kalor, A. E., Stefanovic, C., \& Popovski, P. (2018). Analysis of the communication traffic for blockchain synchronization of iot devices. In 2018 IEEE International Conference on Communications (ICC), p. 1-7. 
[15] Davidson, S., De Filippi, P., \& Potts, J. (2018). Blockchains and the economic institutions of capitalism. Journal of Institutional Economics, 14 (4), p. 639-658.

[16] Gerber, A., Baskerville, R., \& Van der Merwe, A. (2017). A taxonomy of classification approaches in IS research.

[17] Greenspan, G. (2015). Multichain private blockchainwhitepaper.

http://www.multichain.com/download/MultiChain-White-

Paper.pdf [Accessed May 23 ${ }^{\text {rd }}, 2020$ ]

[18] Huawei (2018). Huawei blockchain whitepaper, Huawei Technologies Co., Ltd, p. 1-58.

[19] IBM (2019). IBM Blockchain Platform v.2.1.1. https://cloud.ibm.com/docs/services/blockchain-rhos?topic= blockchain-rhos-get-started-console-ocp [May 23 ${ }^{\text {rd }}, 2020$ ]

[20] Kafka (2017). Apache Kafka. https://kafka.apache.org/ [Accessed May 23 ${ }^{\text {rd }}, 2020$ ]

[21] Kaleido (2019). What is Kaleido?, https://docs.kaleido.io/kaleido-platform/foundationalconcepts/ [Accessed May 23 ${ }^{\text {rd }}, 2020$ ]

[22] Kothari, C. (2004). Research methodology. New Delhi: New Age International (P) Ltd., Publishers.

[23] Labazova, O., Dehling, T., \& Sunyaev, A. (2019). From hype to reality: A taxonomy of blockchain applications. In Proceedings of the 52nd Hawaii International Conference on System Sciences (HICSS 2019).

[24] Lacity, M. C., (2018). Addressing key challenges to making enterprise blockchain applications a reality. MIS Quarterly Executive, 17 (3), p.201-222.

[25] Microsoft (2019). What is Azure Blockchain Workbench? https://docs.microsoft.com/enus/azure/blockchain/workbench/overview [Accessed May $\left.23^{\text {rd }}, 2020\right]$

[26] Morgan, J. P. (2016). Quorum whitepaper. JP Morgan Chase, New York.

[27] Nakamoto, S. (2019). Bitcoin: A peer-to-peer electronic cash system. Manubot.

[28] Nickerson, R.C., Varshney, U., \& Muntermann, J. (2013). A method for taxonomy development and its application in information systems, European Journal of Information Systems, 22 (3), p. 336-359.

[29] Notheisen, B., Hawlitschek, F., \& Weinhardt, C. (2017). Breaking down the blockchain hype-towards a blockchain market engineering approach. Twenty-Fifth European Conference on Information Systems (ECIS), Guimarães, Portugal, p. 1062-1080.
[30] Ongaro, D., \& Ousterhout, J. (2014). In search of an understandable consensus algorithm. In 2014 USENIX Annual Technical Conference ATC, p. 305-319.

[31] Oracle (2020). Using Oracle Blockchain Platform. https://docs.oracle.com/en/cloud/paas/blockchaincloud/user/what-do-i-get-oracle-blockchain-cloud-service. [Accessed May 23 ${ }^{\text {rd }}, 2020$ ]

[32] Pelz-Sharpe, A. (2019). Enterprise Blockchain - Market forecast \& scenarios 2019-2024. Deep Analysis. https://www.deep-analysis.net/wp-content/uploads/2019/08/ DA-190812-Ent-Blockchain-forecast.pdf [May 23 ${ }^{\text {rd }}$, 2020]

[33] Pei, Y., Liu, H., Gao, M., Yang, T., Gao, Q., ... \& Wangzhang, L. (2018). Baidu blockchain whitepaper V1.0. https://view2.doc.nears.cn/doc/201/199/264/e888109eb0e36b 71b4936570d192a98f/index.html?filename=\%E7\%99\%BE\% E5\%BA\%A6\%E5\%8C\%BA\%E5\%9D\%97\%E9\%93\%BE\%E 7\%99\%BD\%E7\%9A\%AE\%E4\%B9\%A6.pdf (originally in Mandarin - translated by Google Translate) [May 23 ${ }^{\text {rd }}, 2020$ ]

[34] Samsung (2018). Nexledger Universal.

https://www.samsungsds.com/global/en/solutions/off/nexledg er/Nexledger.html [Accessed May 23 ${ }^{\text {rd }}, 2020$ ]

[35] SAP (2019). Blockchain applications and services. https://www.sap.com/products/leonardo/blockchain.html?infl =31c6e1a7-da01-4eaf-9153-9ae8f7a70d64 [Accessed May $23^{\text {rd }}, 2020$ ]

[36] Singh, J., \& Michels, J. D., (2018). Blockchain as a Service (BaaS): Providers and trust, 2018 IEEE European Symposium on Security and Privacy Workshops.

[37] Trew, C., Brandon, G., \& Dorier, N. (2019). Stratis Blockchain Solutions whitepaper, Stratis Group Ltd., p. 1-20. https://stratisplatform.com/files/Stratis_Whitepaper.pdf

[38] Tencent (2018). TrustSQL. https://trustsql.qq.com/ [Accessed May $\left.23^{\text {rd }}, 2020\right]$

[39] Weill, P. (2004). Don't just lead, govern: How topperforming firms govern IT. MIS Quarterly Executive, 3 (1), p. 1-17.

[40] Weinhardt, C., \& Gimpel, H. (2007). Market engineering: An interdisciplinary research challenge. In Dagstuhl seminar proceedings. Schloss Dagstuhl-Leibniz-Zentrum für Informatik, p. 2-15.

[41] Yee, J. \& Bremner, C. (2011). Methodological bricolage: What does it tell us about design? In: Doctoral Design Education Conference, 23-25 May 2011, Hong Kong Polytechnic, Hong Kong.

[42] Zhang, J. (2018). Consensus algorithms: PoA, IBFT or Raft? https://kaleido.io/consensus-algorithms-poa-ibft-orraft/. [Accessed May 23 ${ }^{\text {rd }}, 2020$ ]

[43] Zheng, Z., Dai, H., \& Xie, S. (2018). Blockchain challenges and opportunities: A survey, International Journal of Web and Grid Services, 14 (4), p. 352-375. 